\title{
Exact and quasiexact solvability of second order superintegrable quantum systems. II. Relation to separation of variables
}

\author{
E. G. Kalnins \\ Department of Mathematics, University of Waikato, Hamilton, 3240, New Zealand \\ W. Miller, Jr. ${ }^{\text {a) }}$ \\ School of Mathematics, University of Minnesota, Minneapolis, Minnesota 55455 \\ G. S. Pogosyan ${ }^{\text {b) }}$ \\ Departamento de Matématicas, CUCEI, Universidad de Guadalajara, Guadalajara, \\ Jalisco, C. P. 44100, México
}

(Received 23 October 2006; accepted 4 December 2006; published online 2 February 2007)

\begin{abstract}
We make explicit the intimate relationship between quasiexact solvability, as expounded, for example, by Ushveridze [Quasi-exactly Solvable Models in Quantum Mechanics (IOP, Bristol, 1993)], and the technique of separation of variables as it applies to specific superintegrable quantum Hamiltonians. It is the multiseparability of superintegrable systems that forces the existence of interesting families of polynomial solutions characteristic of quasiexact solvability that enables us to solve these systems in distinct ways and that gives us the basis of a classification theory. This connection is generalized in terms of the understanding of the role of finite solutions of quantum Hamiltonians. (C) 2007 American Institute of Physics.
\end{abstract}

[DOI: 10.1063/1.2436733]

\section{INTRODUCTION}

This paper is an extension of our study of the intimate connections between second order superintegrable quantum systems and quasiexact solvability. ${ }^{1}$ It also relies on the structure and classification theory for these systems, as presented in many articles. ${ }^{2-11}$

Here we understand a quasiexactly solvable (QES) problem, in the sense of Turbiner and Ushveridze (in the previous article ${ }^{1}$ we introduced another definition of quasiexact solvability), ${ }^{12}$ as a one dimensional Schrödinger many parameter eigenvalue problem, where for certain values of the parameters, a finite subset of the energy eigenvalues and corresponding eigenfunctions may be found as solutions of algebraic equations. This means in practice that for these fixed parameters, it is possible to write the solution as a special factor times a polynomial and to solve algebraic equations to find the energy one dimensional oscillator with the same anharmonicity. Magyari ${ }^{13}$ stated the theorem that the one dimensional Schrödinger equation

$$
\frac{\mathrm{d}^{2} \psi(x)}{\mathrm{d} x^{2}}+\left[E-V^{(N)}(x)\right] \psi(x)=0,
$$

with anharmonic potential

\footnotetext{
${ }^{a)}$ Electronic mail: mille003@umn.edu

${ }^{b}$ On leave from the Laboratory of Theoretical Physics, JINR, Dubna, Moscow Region 141980, Russia.
} 


$$
V^{(N)}(x)=\sum_{n=1}^{2 N-1} b_{n} x^{2 n}, \quad b_{2 N-1}>0, \quad N=1,2,3 \ldots,
$$

admits square integrable even $(s=0)$ and odd $(s=1)$ parity eigenfunctions of the form

$$
\psi^{(s)}(x)=\exp \left(-\frac{1}{2} \sum_{n=1}^{N} a_{n} x^{2 n}\right) x^{s} P_{M}^{(s)}(x), \quad a_{N}>0
$$

where

$$
P_{M}^{(s)}(x)=\sum_{m=0}^{M} c_{m}^{(s)} x^{2 m}
$$

is a polynomial of degree $M, M=0,1,2 \ldots$, if the first parameters $\left(b_{1}, b_{2}, \ldots, b_{N-1}\right)$ satisfy a set of $N-1$ constraints. The last constrained parameter $b_{N-1}$ is given by the following formula:

$$
b_{N-1}=-N(2 N+4 M+2 s-1) a_{N}+\sum_{i, j}^{i+j=N} i j a_{i} a_{j}
$$

which ensures the existence of a polynomial solution of degree $2 M$, Eq. (4).

The numbers $a_{i}(i=1,2, \ldots, N)$ formed from the $N$ arbitrary constants $\left(b_{N}, b_{N+1}, \ldots, b_{2 N-1}\right)$; the energy $E$, the coefficients $c_{m}^{(s)}$, and constrained coupling constants $\left(b_{1}, b_{2}, \ldots, b_{N-1}\right)$ are determined from the systems of algebraic equations (see for details Ref. 13).

The change of variable $|y|=x^{2}$ or $x=\operatorname{sgn} y \sqrt{|y|}(\mathbf{R} \rightarrow \mathbf{R})$ together with a wave function transformation $\psi(y)=|y|^{-1 / 4} \phi(y)$ reduces Eq. (1) to

$$
\frac{\mathrm{d}^{2} \phi(y)}{\mathrm{d} y^{2}}+\left[\epsilon+\frac{\alpha}{|y|}-\frac{\nu(\nu-1)}{y^{2}}-\widetilde{V}^{(N)}(y)\right] \phi(y)=0,
$$

where $\epsilon=-b_{1} / 4, \alpha=E / 4, \nu$ takes two values $\nu=1 / 4, \nu=3 / 4$, and

$$
\tilde{V}^{(N)}(y)=\frac{1}{4}\left(b_{2} y+b_{3} y^{2}+\cdots+b_{2 N-1} y^{2 N-2}\right)=\frac{1}{4} \sum_{n=1}^{2 N-2} b_{n+1} y^{n}, \quad N=2,3, \ldots
$$

and gives us a new class of QES systems which are dual to the first one. Indeed, in Eq. (6) the energy $E$ is fixed and plays the role of a coupling constant, the coupling constant $b_{1}$ is quantized, and the quantity $\epsilon=-b_{1} / 4$ has the meaning of energy. Note also that after the substitution $y=x^{2}$ $>0\left(\mathbf{R} \rightarrow \mathbf{R}^{+}\right)$and with $\psi(y)=y^{-1 / 4} \phi(y)$, Eq. (1) transforms to an equation having the same form as Eq. (6) but where $y$ has the meaning of a radial variable for the three dimensional Schrödinger equation.

Recently, an interesting extension of the QES family has been obtained in the article of Bender and Boettchter. ${ }^{14}$ They introduced a new class of QES $\mathcal{P} \mathcal{T}$ symmetric Hamiltonians (see also Ref. 15 for $\mathcal{P} \mathcal{T}$ symmetric quantum mechanics) with a quartic polynomial potential, which formally can be considered as a particular case of Eq. (6) for $N=2, \alpha=0, \nu=0$, and imaginary coupling coefficients $b_{2}$ and $b_{4}$. The further generalization of QES systems in the complex plane is presented in articles of Znojil. ${ }^{16-18}$

The main problem in subsequent investigations of QES systems is to understand just which potentials and just which specializations of the parameters in these potentials allow explicit algebraic solutions.

It has long been understood ${ }^{12,19}$ that one way of approaching such problems is to obtain the eigenfunction equation as one of the separation equations for a higher dimensional [partial differ- 
ential equation (PDE)] Schrödinger equation that is separable in some set of coordinates. The parameters are the separation constants, and the permissible values of these parameters and energy eigenvalues are obtained by solving the PDE eigenvalue problem.

In our work we make clear that QES equations are better treated as arising from higher dimensional superintegrable systems, in particular, those that are multiseparable. ${ }^{1}$ This enables us to solve these systems in distinct ways, thus better understanding the solutions, and gives us the basis of a classification theory. ${ }^{10,20}$ Our approach leads us to many new examples of QES systems.

To motivate our approach we start by reviewing some simple exactly solvable (ES) and QES systems. The solution of many of the PDEs of mathematical physics can be achieved via the method of separation of variables. Among the solutions found in this way are classical polynomials such as those of Legendre, Laguerre, Hermite, and Jacobi. ${ }^{21}$ As an example, Legendre polynomials $P_{\ell}(x)$ satisfy the differential equation

$$
\left(1-x^{2}\right) \frac{\mathrm{d}^{2}}{\mathrm{~d} x^{2}} P_{\ell}(x)-2 x \frac{\mathrm{d}}{\mathrm{d} x} P_{\ell}(x)+\ell(\ell+1) P_{\ell}(x)=0, \quad-1 \leqslant x \leqslant 1 .
$$

If we look for polynomial solutions $\Pi_{i=1}^{\ell}\left(x-\theta_{i}\right)$ of this equation we find as necessary and sufficient conditions that the zeros $\theta_{i}$ must satisfy the relation

$$
(2 \ell+1) \theta_{i}+2 \sum_{j \neq i} \frac{1}{\theta_{j}-\theta_{i}}=0 .
$$

These are just the relations satisfied by the zeros of the Legendre polynomials $P_{\ell}(x)$. Now consider the Laplace equation

$$
\left(\frac{\partial^{2}}{\partial x^{2}}+\frac{\partial^{2}}{\partial y^{2}}+\frac{\partial^{2}}{\partial z^{2}}\right) f=0
$$

expressed in conical coordinates $u, v, r\left(r>0, e_{1}<u<e_{2}<v<e_{3}\right)$,

$$
x^{2}=r^{2} \frac{\left(u-e_{1}\right)\left(v-e_{1}\right)}{\left(e_{1}-e_{2}\right)\left(e_{1}-e_{3}\right)}, \quad y^{2}=r^{2} \frac{\left(u-e_{2}\right)\left(v-e_{2}\right)}{\left(e_{2}-e_{1}\right)\left(e_{2}-e_{3}\right)}, \quad z^{2}=r^{2} \frac{\left(u-e_{3}\right)\left(v-e_{3}\right)}{\left(e_{3}-e_{1}\right)\left(e_{3}-e_{2}\right)} .
$$

If we look for separated solutions of the form $f=r^{\ell} U(u) V(v)$, we see that the separation equations for the functions $U(u)$ and $V(v)$ are of Lamé type, viz.,

$$
\sqrt{P(\lambda)} \frac{\mathrm{d}}{\mathrm{d} \lambda} \sqrt{P(\lambda)} \frac{\mathrm{d}}{\mathrm{d} \lambda} \Lambda(\lambda)+[-\ell(\ell+1) \lambda+\mu] \Lambda(\lambda)=0,
$$

where $P(x)=-4\left(x-e_{1}\right)\left(x-e_{2}\right)\left(x-e_{3}\right), \Lambda=U, V$, and $\lambda=u, v$. Requiring polynomial solution

$$
\Lambda(\lambda)=\left(\lambda-e_{1}\right)^{p}\left(\lambda-e_{2}\right)^{q}\left(\lambda-e_{3}\right)^{r} \Pi_{i=1}^{n}\left(\lambda-\theta_{i}\right),
$$

where $p, q, r$ are 0 or $\frac{1}{2}$, we see that this is possible if and only if

$$
\frac{p+1}{\theta_{i}-e_{1}}+\frac{q+1}{\theta_{i}-e_{2}}+\frac{r+1}{\theta_{i}-e_{3}}+\sum_{j \neq i} \frac{2}{\theta_{i}-\theta_{j}}=0
$$

where $\ell=2(n+p+q+r)$ and

$$
\begin{aligned}
\mu= & e_{1}[-4(r+q+n) 2+2 p(2 p-1)]+e_{2}[-4(r+p+s) 2+2 q(2 q-1)] \\
& +e_{3}[-4(p+q+n) 2+2 r(2 r-1)]+[8(p+q+r+n)-2] \sum_{i=1}^{n} \theta_{i} .
\end{aligned}
$$

Such polynomial solutions can also be obtained for the Schrödinger equation $H \Psi=E \Psi$ in the presence of a potential. An example of this in three dimensions ${ }^{22}$ is 


$$
H=-\left(\frac{\partial^{2}}{\partial x^{2}}+\frac{\partial^{2}}{\partial y^{2}}+\frac{\partial^{2}}{\partial z^{2}}\right)+\left[\omega^{2}\left(x^{2}+y^{2}+z^{2}\right)+\frac{p^{2}-1 / 4}{x^{2}}+\frac{q^{2}-1 / 4}{y^{2}}+\frac{r^{2}-1 / 4}{z^{2}}\right] .
$$

If we again choose conical coordinates and look for solutions of the form $\Psi=R(r) U(u) V(v)$, we see that the functions $U(u)$ and $V(v)$ must satisfy the differential equation

$$
\begin{gathered}
\sqrt{P(\lambda)} \frac{\mathrm{d}}{\mathrm{d} \lambda}\left(\sqrt{P(\lambda)} \frac{\mathrm{d}}{\mathrm{d} \lambda} \Lambda(\lambda)\right)+\left[-\ell(\ell+1) \lambda+\mu+\left(e_{1}-e_{2}\right)\left(e_{1}-e_{3}\right) \frac{\left(p^{2}-1 / 4\right)}{\lambda-e_{1}}\right. \\
\left.+\left(e_{2}-e_{1}\right)\left(e_{2}-e_{3}\right) \frac{\left(q^{2}-1 / 4\right)}{\lambda-e_{2}}+\left(e_{3}-e_{2}\right)\left(e_{3}-e_{1}\right) \frac{\left(r^{2}-1 / 4\right)}{\lambda-e_{3}}\right] \Lambda(\lambda)=0,
\end{gathered}
$$

where $\Lambda=U, V$ and $\lambda=u, v$. Looking for solutions in exactly the same manner as we have done for solutions of Laplace's equation in these coordinates, we uncover the same relations obeyed by the zeros $\theta_{i}$ and the same expressions for $\ell$ and $\mu$. The only difference is that $p, q$, and $r$ can now be arbitrary. The radial equation satisfied by $R(r)$ is

$$
\frac{\mathrm{d}^{2} R}{\mathrm{~d} r^{2}}+\frac{2}{r} \frac{\mathrm{d} R}{\mathrm{~d} r}+\left[E-\omega^{2} r^{2}-\frac{\ell(\ell+1)}{r^{2}}\right] R=0 .
$$

This equation admits polynomial solutions of the form

$$
R(r)=r^{\ell} \exp \left(-\frac{\omega}{2} r^{2}\right) L_{n}^{\ell+1 / 2}\left(\omega r^{2}\right),
$$

where the energy eigenvalues are $E_{n}=\omega(2 n+p+q+r+3)$ for $n=0,1, \ldots$ and the zeros of the associated Laguerre polynomials $L_{n}^{\alpha}(z) \approx \prod_{i=1}^{s}\left(z-\theta_{i}\right)$ satisfy the relations

$$
-\alpha-1+\theta_{i}+2 \sum_{j \neq i} \frac{\theta_{i}}{\theta_{j}-\theta_{i}}=0 .
$$

The above examples are all ES systems because the solutions can be given explicitly in terms of hypergeometric functions (for instance, in spherical or Cartesian coordinates).

To motivate our more complicated results to follow, we give one more example, a true QES system. This example is closely related to one treated in our paper ${ }^{1}$ but its ingredients are relevant to the present work. Consider the differential equation

$$
\frac{\mathrm{d}^{2} F}{\mathrm{~d} \ell^{2}}+\left\{-144 k_{1}^{2} \ell^{4}-96 k_{1} k_{2} \ell^{3}+16\left(2 k_{2}^{2}+3 k_{1} k_{3}\right) \ell^{2}+E \ell+\mu\right\} F=0
$$

a form of the triconfluent Heun equation that completely coincides, for $\ell=i x, k_{1}=1 / 12,4 k_{2}=a$, $4 k_{3}=2 b-3 a^{2}, \mu=-E$, and $J=N+1$, with the $\mathcal{P} \mathcal{T}$ symmetric QES example presented in Ref. 14. It is known ${ }^{14,23}$ that in the case of

$$
E=8\left[2 k_{2} k_{3}+2 \frac{k_{2}^{3}}{k_{1}}-3 k_{1}(n+1)\right], \quad n=0,1,2 \ldots,
$$

Eq. (10) has finite solution

$$
F=\exp \left(4 k_{1} \ell^{3}+2 k_{2} \ell^{2}-2\left(\frac{k_{2}^{2}}{k_{1}}+k_{3}\right) \ell\right) \prod_{i=0}^{n}\left(\ell-\theta_{i}\right),
$$

where the $\theta_{i}$ satisfy the relation 


$$
-2 k_{1} k_{3}-2 k_{2}^{2}+4 k_{1} k_{2} \sum_{i=1}^{n} \theta_{i}+12 k_{1}^{2} \sum_{i=1}^{n} \theta_{i}^{2}+\sum_{j \neq i} \frac{k_{1}}{\theta_{i}-\theta_{j}}=0,
$$

and the parameter $\mu$ is determined by the formula

$$
\mu=-4 \frac{k_{2}^{4}}{k_{1}^{2}}-8 \frac{k_{2}^{2} k_{3}}{k_{1}}-4 k_{3}^{2}-4(1+2 n) k_{2}-24 \sum_{i=1}^{n} \theta_{i}
$$

Thus we have a true QES solution for a potential that is a polynomial of order 4. It is also possible to obtain such solutions by factoring out the expression $f=\exp \left(4 k_{1} \ell^{3}+2 k_{2} \ell^{2}-2\left(k_{2}^{2} / k_{1}+k_{3}\right) \ell\right)$ and then looking directly for strictly polynomial solutions (see, for instance, Ref. 14).

A natural question is as follows: How is this particular set of solutions related to superintegrability? To answer this consider the quantum Hamiltonian

$$
\begin{aligned}
H= & \frac{\partial^{2}}{\partial x^{2}}+\frac{\partial^{2}}{\partial y^{2}}+36 k_{1}^{2}\left[2(x-i y)^{3}-4\left(x^{2}+y^{2}\right)\right]+24 k_{1} k_{2}\left[3(x-i y)^{2}-2(x+i y)\right] \\
& -16\left(2 k_{2}^{2}+3 k_{1} k_{3}\right)(x-i y) .
\end{aligned}
$$

The corresponding Schrödinger equation is superintegrable and multiseparable. ${ }^{6}$ If the coordinates $x$ and $y$ are related to semihyperbolic coordinates $u, v$ via

$$
x+i y=-\frac{1}{2}(u-v)^{2}, \quad x-i y=u+v,
$$

then the Schrödinger equation can be written as

$$
\begin{aligned}
H \Psi= & \left\{\frac { 1 } { u - v } \left[\left(\frac{\partial^{2}}{\partial u^{2}}-144 k_{1}^{2} u^{4}-96 k_{1} k_{2} u^{3}+16\left(2 k_{2}^{2}+3 k_{1} k_{3}\right) u^{2}\right)\right.\right. \\
& \left.\left.-\left(\frac{\partial^{2}}{\partial v^{2}}-144 k_{1}^{2} v^{4}-96 k_{1} k_{2} v^{3}+16\left(2 k_{2}^{2}+3 k_{1} k_{3}\right) v^{2}\right)\right]\right\} \Psi=E \Psi .
\end{aligned}
$$

This can be solved for $\Psi$ via the usual separation of variables ansatz $\Psi=U(u) V(v)$. In particular, we can look for finite solutions of the form

$$
\Psi=\exp \left[4 k_{1}\left(u^{3}+v^{3}\right)+2 k_{2}\left(u^{2}+v^{2}\right)-2\left(\frac{k_{2}^{2}}{k_{1}}+k_{3}\right)(u+v)\right] \prod_{i=1}^{n}\left(u-\theta_{i}\right)\left(v-\theta_{i}\right),
$$

where the functions $U(u)$ and $V(v)$ satisfy the same separated differential equation as $F$ above and the energy spectrum given the formula (11).

Another important question is as follows: How can we determine the values of $\mu$ for polynomial solutions of this type? This is done by noting that $\mu$ is the eigenvalue of the symmetry operator

$$
\begin{aligned}
M= & \frac{1}{u-v}\left[v\left(\frac{\partial^{2}}{\partial u^{2}}-144 k_{1}^{2} u^{4}-96 k_{1} k_{2} u^{3}+16\left(2 k_{2}^{2}+3 k_{1} k_{3}\right) u^{2}\right)\right. \\
& \left.-u\left(\frac{\partial^{2}}{\partial v^{2}}-144 k_{1}^{2} v^{4}-96 k_{1} k_{2} v^{3}+16\left(2 k_{2}^{2}+3 k_{1} k_{3}\right) v^{2}\right)\right] .
\end{aligned}
$$

The eigenvalues can be determined by solving the eigenvalue equation $M \Psi=\mu \Psi$ in the form 


$$
\Psi=\exp \left(4 k_{1}\left(u^{3}+v^{3}\right)+2 k_{2}\left(u^{2}+v^{2}\right)-2\left(\frac{k_{2}^{2}}{k_{1}}+k_{3}\right)(u+v)\right) S(u, v),
$$

where $S(u, v)$ is a sum of symmetric polynomials

$$
S_{i j}=S_{j i}=u^{i} v^{j}+u^{j} v^{i}, \quad 0 \leqslant i+j \leqslant 2 n,
$$

i.e., $S(u, v)=\sum_{i \leqslant j} a_{i j} S_{i j}$, subject to the restriction $0 \leqslant i+j \leqslant 2 n$. There are $\frac{1}{2}(n+1)(n+2)$ such functions. If we require that $M \Psi=\mu \Psi$ then we must solve a set of linear equations of the form

$$
\sum_{k \leqslant \ell}\left(C_{i j}^{k \ell}-\mu\right) a_{k \ell}=0
$$

where

$$
\hat{M} S_{i j}=\sum_{k \leqslant \ell} C_{i j}^{k \ell} S_{k \ell}
$$

and $\hat{M}$ is the induced action of $M$ on $S(u, v)$. There is a determinantal condition to be satisfied in order that these equations have nontrivial solutions. This condition must include the possibility of solutions of the form

$$
\Psi=\exp \left[4 k_{1}\left(u^{3}+v^{3}\right)+2 k_{2}\left(u^{2}+v^{2}\right)-2\left(\frac{k_{2}^{2}}{k_{1}}+k_{3}\right)(u+v)\right] \Pi_{i=1}^{s}\left(u-\theta_{i}\right)\left(v-\theta_{i}\right),
$$

where $0 \leqslant s \leqslant n$. This is clear from the observation that we have a linear system of dimension $\frac{1}{2}(n+1)(n+2)$. If we wish to determine the eigenvalues associated with solutions corresponding to $s=n$ only then we could take $S_{n n}=1$. The rank of the system then becomes $n+1$, but the price we pay for this is that the determinantal condition is more awkward to implement. As an illustration of this process, if we look for solutions of the form

$$
S(u, v)=c_{1} u v+c_{2}(u+v)+c_{3},
$$

then $\mu$ satisfies the factorizable cubic equation

$$
\begin{gathered}
\left(\mu k_{1}^{2}+4 k_{1}^{2} k_{3}^{2}+4 k_{2} k_{1}^{2}+4 k_{2}^{4}+8 k_{1} k_{3} k_{2}^{2}\right)\left(k_{1}^{4} \mu^{2}+8 k_{1}^{2}\left(2 k_{2} k_{1}^{2}+k_{3}^{2} k_{1}^{2}+2 k_{1} k_{3} k_{1}^{2}+k_{2}^{4}\right) \mu-96 k_{3} k_{1}^{5}-48 k_{2}^{2} k_{1}^{2}\right. \\
\left.+64 k_{3}^{2} k_{1}^{2} k_{2}+16 k_{3}^{4} k_{1}^{4}+128 k_{2}^{3} k_{3} k_{1}^{3}+64 k_{3}^{3} k_{1}^{3} k_{2}^{2}+64 k_{2}^{5} k_{1}^{2}+96 k_{1}^{2} k_{2}^{4} k_{3}^{2}+64 k_{2}^{6} k_{1} k_{3}+16 k_{2}^{8}\right)=0 .
\end{gathered}
$$

Thus we have used the superintegrable system to determine the values $\mu, E$ for which the separated equation is QES, and we have obtained the results in an alternate from that for the separated equations treated alone. Note further that if we perform a special ansatz in the nonseparable Cartesian coordinates to try to find polynomial solutions in $x, y$, we will obtain exactly the same exponential phase factor as the product of the phase factors in $u$ and in $v$. This means that the possible polynomial solutions in $x, y$ for a fixed energy eigenspace [Eq. (11)] correspond exactly with the polynomial solutions symmetric in $u$ and $v$. The polynomial energy eigenspace is degenerate but the diagonalization of $M$ breaks the degeneracy.

We can exploit the fact that our superintegrable system is multiseparable. It also separates in shifted semihyperbolic coordinates obtained formally from the standard semihyperbolic coordinates [Eq. (12)] through the transformation

$$
x \rightarrow x+a, \quad y \rightarrow y-i a .
$$

Then

$$
V(x, y)-E \rightarrow V(x, y)-96 a k_{1}\left[3 k_{1}(x-i y)-k_{2}\right]-E=\bar{V}(x, y)-\bar{E},
$$

which is again of the same form if we make the transformations 


$$
k_{1} \rightarrow k_{1}, \quad k_{2} \rightarrow k_{2}, \quad k_{3} \rightarrow 6 a k_{1}+k_{3}, \quad E \rightarrow E+96 k_{1} k_{2} a
$$

of the parameters. From this observation we deduce that separation of variables can also be achieved in displaced coordinates for arbitrary $a$. Also the polynomial solutions in the $u, v$ coordinates can be written as linear combinations of the polynomials in the shifted separable coordinates.

In addition to the possibility of solving Schrödinger's equation via separation of variables, there is the possibility of obtaining an explicit solution that corresponds to the Lie form in the corresponding classical mechanical problem. This form can be calculated by writing the Schrödinger equation in complex conjugate coordinates, in which it becomes

$$
\frac{\partial^{2} \Psi}{\partial z \partial \bar{z}}+(A(\bar{z}) z+B(\bar{z})) \Psi=0,
$$

not separable but solvable. We set $\Psi=\mathrm{e}^{z C(\bar{z})+D(\bar{z})}$. This will be a solution provided that

$$
C(\bar{z})^{2}=2 \int A(\bar{z}) \mathrm{d} \bar{z}, \quad D(\bar{z})=\int \frac{B(\bar{z})}{C(\bar{z})} \mathrm{d} \bar{z}-\ln C(\bar{z}) .
$$

In our case we obtain the solution

$$
\begin{gathered}
C(\bar{z})=2 \sqrt{3 k_{1}}\left(3 k_{1} \bar{z}^{2}+2 k_{2} \bar{z}+\Lambda\right)^{1 / 2}, \\
D(\bar{z})=-\frac{1}{8 \sqrt{3 k_{1}}}\left[\frac{1}{3}\left(-16 k_{1}^{3} \Lambda+52 k_{1}^{2} k_{2} \bar{z}-84 k_{1}^{2} k_{2}^{2}-48 k_{1}^{2} k_{3}+24 k_{1}^{4} \bar{z}^{2}\right)\left(3 k_{1} \bar{z}^{2}+2 k_{2} \bar{z}+\Lambda\right)^{1 / 2}\right. \\
+\frac{1}{k_{1}^{2} \sqrt{3 k_{1}}}\left(28 k_{2}^{3}-12 k_{1} k_{2} \Lambda-E k_{1}+16 k_{1} k_{2} k_{3}\right) \ln \left[2 \sqrt{3 k_{1}}\left(3 k_{1} \bar{z}+k_{2}\right)\right. \\
\left.\left.+6 k_{1}\left(3 k_{1} \bar{z}^{2}+2 k_{2} \bar{z}+\Lambda\right)^{1 / 2}\right]\right]-\frac{1}{2} \ln \left(3 k_{1} \bar{z}^{2}+2 k_{2} \bar{z}+\Lambda\right)
\end{gathered}
$$

to within a constant. Can $\Lambda$ and $E$ be chosen in such a way that there are "polynomial" solutions to within a factor of this type? We note here that the constant $\Lambda$ has a definite meaning in terms of symmetries of $H$. One could in principle expand a given set of such solutions in terms of a separable set of solutions, but it is our intention to study this elsewhere.

Based on these examples, a natural question to ask is as follows: What is the most general way that we can obtain one dimensional (1D) QES eigenvalue problems and their solutions, related to separation of variables of a nondegenerate superintegrable system?

\section{POLYNOMIAL SOLUTIONS FROM EUCLIDEAN SPACE SUPERINTEGRABLE SYSTEMS}

A crucial observation to answer the preceding question is that the Hamiltonian $H$ in our example is superintegrable and admits three second order symmetry operators: a quantum superintegrable system. To answer the question fully we need to recall some facts about generic elliptical coordinates in complex Euclidean $n$ space and on the complex $n$ sphere, and their relationship to superintegrable systems with nondegenerate potentials.

A quantum system of the form $H \Psi=E \Psi$, where $H=\Delta_{n}+V(\mathbf{x})$ (and $\Delta_{n}$ is the Laplace-Beltrami operator) on an $n$ dimensional real or complex pseudo-Riemannian manifold is second order superintegrable if there are $2 n-1$ second order differential operators (expressed in local coordinates $\left.x_{s}\right), L_{j}=\Sigma_{k, \ell} a_{(j)}^{k \ell} \partial_{k \ell}^{2}+$ lower order terms, $j=1, \ldots, 2 n-1$, that commute with $H=L_{1}$ and such that the corresponding quadratic forms $\mathcal{L}_{j}=\Sigma_{k, \ell} a_{(j)}^{k \ell} p_{k} p_{\ell}$ are functionally independent on an open set in the classical $2 n$ dimensional phase space. In general, the potential $V$ and the corresponding potential terms in the symmetry operators $L_{j}$ need not be fixed but can range over a vector space 
of potentials. Suppose that the pseudo-Riemannian space is conformally flat and the $x_{s}$ are Cartesian-like coordinates with respect to which the metric tensor takes the diagonal form $g^{k \ell}$ $=\delta^{k \ell} / \lambda(\mathbf{x})$. The potential is nondegenerate if it is the general solution of a system of the form

$$
\begin{gathered}
V_{j j}-V_{11}=\sum_{\ell=1}^{n} A^{j j, \ell}(\mathbf{x}) V_{\ell}, \quad j=2, \ldots, n, \\
V_{k j}=\sum_{\ell=1}^{n} A^{k j, \ell}(\mathbf{x}) V_{\ell}, \quad 1 \leqslant k<j \leqslant n,
\end{gathered}
$$

where all of the integrability conditions for this system of PDEs are identically satisfied. ${ }^{8,9}$ (Here $V_{j}=\partial_{j} V$, etc., and the $A^{k j, \ell}$ are analytic in an open set of the $n$ dimensional space with coordinates $\mathbf{x}$.) In this case there is an $n+2$ dimensional solution space of nondegenerate potentials $V$ that can be parametrized by choosing the values $V\left(\mathbf{x}_{0}\right), V_{11}\left(\mathbf{x}_{0}\right), V_{\ell}\left(\mathbf{x}_{0}\right), \ell=1, \ldots, n$ at some fixed point $\mathbf{x}_{0}$. One ordinarily refers to these nondegenerate potentials as $n+1$ parameter, neglecting the trivial constant that can be added to any potential. Equations (13) always arise from the BertrandDarboux equations for the potential of a superintegrable systems such that the $2 n-1$ symmetries are linearly independent at a point, ${ }^{9}$ but in general the integrabilty conditions are not satisfied identically so that the space of solutions $V$ has dimension $<n+2$. Thus the nondegenerate potentials are those with the maximum number of parameters possible.

Classification of the possible superintegrable systems with nondegenerate potentials in complex Euclidean $n$-space is a very difficult task that has been carried out only for $n=2$, with significant results for $n=3 .{ }^{8,10}$ However, there is an important subclass of such systems that can be constructed for all $n \geqslant 2$, based on their relationship to variable separation in generic Jacobi elliptic coordinates. The prototype superintegrable system which is nondegenerate in $n$ dimensional flat space has the Hamiltonian ${ }^{22}$

$$
H=\sum_{i=1}^{n}\left(\partial_{i}^{2}+\alpha x_{i}^{2}+\frac{\beta_{i}}{x_{i}^{2}}\right)+\delta, \quad \partial_{i}=\partial_{x_{i}} .
$$

The system $H \Psi=E \Psi$ is superintegrable with nondegenerate potential and a basis of $n(n+1) / 2$ second order symmetry operators given by

$$
P_{i}=\partial_{i}^{2}+\alpha x_{i}^{2}+\frac{\beta_{i}}{x_{i}^{2}}, \quad J_{i j}=\left(x_{i} \partial_{j}-x_{j} \partial_{i}\right)^{2}+\beta_{i} \frac{x_{j}^{2}}{x_{i}^{2}}+\beta_{j} \frac{x_{i}^{2}}{x_{j}^{2}}, \quad i \neq j .
$$

Although there appear to be "too many" symmetry operators, all are functionally dependent on a subset of $2 n-1$ functionally independent symmetries. A crucial observation is that the corresponding equation $H \Psi=E \Psi$ admits multiplicative separation in $n$ generic elliptical coordinates $x_{i}^{2}$ $=c^{2} \Pi_{j=1}^{n}\left(u_{j}-e_{i}\right) / \Pi_{k \neq i}\left(e_{k}-e_{i}\right)$, simultaneously for all values of the parameters with $e_{i} \neq e_{j}$ if $i \neq j$ and $i, j=1, \ldots, n$. If we were dealing with real Euclidean space then we could assume that the inequalities $e_{1}<e_{2}<\cdots<e_{n}$ and $e_{1}<u_{1}<e_{2}<\cdots<e_{n}<u_{n}$ hold. Thus the equation is multiseparable and separates in a continuum of elliptic coordinate systems (and in many others besides). The $n$ commuting symmetries characterizing a fixed elliptic separable system are polynomial functions of the $e_{i}$, and requiring separation for all $e_{i}$ simultaneously sweeps out the full $n(n$ $+1) / 2$ space of symmetries and uniquely determines the nondegenerate potential. The infinitesimal distance in Jacobi elliptical coordinates $u_{j}$ has the form

$$
\mathrm{d} s^{2}=-\frac{c^{2}}{4} \sum_{i=1}^{n} \frac{\Pi_{j \neq i}\left(u_{i}-u_{j}\right)}{P\left(u_{i}\right)} \mathrm{d} u_{i}^{2},
$$

where $P(\lambda)=\prod_{k=1}^{n}\left(\lambda-e_{k}\right)$. However, it is well known that Eq. (15) is a flat space metric for any polynomial $P(\lambda)$ of order $\leqslant n$ and that each choice of such a $P(\lambda)$ defines an elliptic-type multi- 
plicative separable solution of the Laplace-Beltrami eigenvalue problem (with constant potential) in complex Euclidean $n$-space. ${ }^{24}$ The distinct cases are labeled by the degree of the polynomial and the multiplicities of its distinct roots. If for each distinct case we determine the most general potential that admits separation for all $e_{i}$ compatible with the multiplicity structure of the roots, we obtain a unique superintegrable system with nondegenerate potential and $n(n+1) / 2$ second order symmetries. ${ }^{10,20}$ These are the generic complex superintegrable systems. [Thus, for $n=3$ there are seven distinct cases for $-\frac{1}{4} P(\lambda)$ :

$$
\begin{gathered}
\left(\lambda-e_{1}\right)\left(\lambda-e_{2}\right)\left(\lambda-e_{3}\right), \quad\left(\lambda-e_{1}\right)\left(\lambda-e_{2}\right)^{2}, \quad\left(\lambda-e_{1}\right)^{3}, \\
\left(\lambda-e_{1}\right)\left(\lambda-e_{2}\right), \quad\left(\lambda-e_{1}\right)^{2}, \quad\left(\lambda-e_{1}\right), \quad 1,
\end{gathered}
$$

where $e_{i} \neq e_{j}$ for $i \neq j$. The first case corresponds to Jacobi elliptic coordinates.] The number of distinct generic superintegrable systems for each integer $n \geqslant 2$ is

$$
\sum_{j=0}^{n} p(j)
$$

where $p(j)$ is the number of integer partitions of $j$, given by the Euler generating function

$$
\frac{1}{\Pi_{k=1}^{\infty}\left(1-t^{k}\right)}=\sum_{j=0}^{\infty} p(j) t^{j} .
$$

All of the generic separable systems, their potentials, and their defining symmetries can be obtained from the basic Jacobi elliptic system in $n$ dimensions by a complicated but well defined set of limit processes. ${ }^{10,20}$ Although we cannot write down master canonical expressions for all such generic systems in Cartesian coordinates, it is easy to take these limits and write down a master equation for the separated ordinary differential equations in the elliptic coordinates, $u_{i}$. The separation equation for each of the coordinates $x=u_{i}$ is essentially the same,

$$
\begin{gathered}
\sqrt{P(x)} \frac{\mathrm{d}}{\mathrm{d} x}\left(\sqrt{P(x)} \frac{\mathrm{d} F}{\mathrm{~d} x}\right)+\left(a_{n+p_{0}} x^{n+p_{0}}+\cdots+a_{n} x^{n}+\hat{a}_{n-1} x^{n-1}+\cdots+\hat{a}_{0}+\frac{b_{1}^{(1)}}{\left(x-e_{1}\right)}+\frac{b_{2}^{(1)}}{\left(x-e_{1}\right)^{2}}\right. \\
\left.+\cdots+\frac{b_{p_{1}}^{(1)}}{\left(x-e_{1}\right)^{p_{1}}}+\cdots+\frac{b_{1}^{(r)}}{\left(x-e_{r}\right)}+\frac{b_{2}^{(r)}}{\left(x-e_{r}\right)^{2}}+\cdots+\frac{b_{p_{r}}^{(r)}}{\left(x-e_{r}\right)^{p_{r}}}\right) F=0, \\
P(x)=-4\left(x-e_{1}\right)^{p_{1}} \cdots\left(x-e_{r}\right)^{p_{r}},
\end{gathered}
$$

and $p_{0}+p_{1}+\cdots+p_{r}=n$. Here the $n$ constants $\hat{a}_{s}, s=0, \ldots, n-1$, are the separation constants for the superintegrable system in these elliptic coordinates. In particular, $E=\hat{a}_{n-1}$. The other $n+1$ constants depend on the $n+1$ parameters in the potential and can be assigned arbitrarily by specifying the appropriate potential.

First we look for explicit solutions of the single equation [Eq. (16)], ignoring the fact that it is a separation equation for a superintegrable system. To obtain polynomials, we perform a gauge transformation and look for solutions of the form 


$$
\begin{aligned}
& \Psi=\exp \left(\ell_{1} x+\ell_{2} x^{2}+\cdots+\ell_{p_{0}+1} x^{p_{0}+1}+\frac{c_{1}^{(1)}}{\left(x-e_{1}\right)}+\frac{c_{2}^{(1)}}{\left(x-e_{1}\right)^{2}}+\cdots+\frac{c_{p_{1}-1}^{(1)}}{\left(x-e_{p_{1}}\right)^{p_{1}-1}}+\cdots+\frac{c_{1}^{(r)}}{\left(x-e_{r}\right)}\right.
\end{aligned}
$$

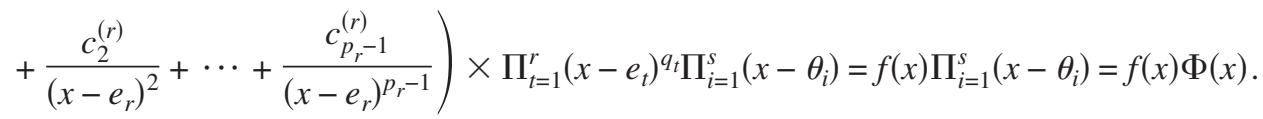

We require that the differential equation satisfied by $\Phi(x)$ is of the form

$$
\left(r_{n-p_{0}} x^{n-p_{0}}+\cdots+r_{0}\right) \frac{\mathrm{d}^{2} \Phi}{\mathrm{d} x^{2}}+\left(s_{n} x^{n}+\cdots+s_{0}\right) \frac{\mathrm{d} \Phi}{\mathrm{d} x}+\left(t_{n-1} x^{n-1}+t_{0}\right) \Phi=0 .
$$

This can be achieved by choosing values for $a_{n+p_{0}}, \ldots, a_{n}$ to make the coefficients of powers of $x\left(x^{i}, i=n+p_{0}, \ldots, n\right)$ zero in the expression for the polynomial multiplying $\Phi$ in Eq. (19). Similarly by choosing the coefficients $b_{j}^{(J)} j=1, \ldots, p_{J}$, all singular terms in this equation can be removed. Now, polynomial solutions of Eq. (19) can be sought and there are just enough conditions to determine $\hat{a}_{n-1}, \ldots, \hat{a}_{0}$ and $\theta_{i}, i=1, \ldots, s$, in terms of a solution $\Phi=\Pi_{i=1}^{s}\left(x-\theta_{i}\right)$. We will not write down these equations for the separation constants in the general case, but note that they exist. The zeros $\theta_{i}$ of these polynomials satisfy relations of the form

$$
\begin{gathered}
\sum_{j \neq i} \frac{1}{\theta_{i}-\theta_{j}}+\ell_{1} \theta_{i}+2 \ell_{2} \theta_{i}^{2}+\cdots+\left(p_{0}+1\right) \ell_{p_{0}+1} \theta_{i}^{p_{0}}+\frac{1 / 4 p_{1}+q_{1}}{\left(\theta_{i}-e_{1}\right)}-\frac{c_{1}^{(1)}}{\left(\theta_{i}-e_{1}\right)^{2}} \\
\quad+\frac{\left(p_{1}-1\right) c_{p_{1}-1}^{(1)}}{\left(\theta_{i}-e_{1}\right)^{p_{1}}} \cdots+\frac{1 / 4 p_{r}+q_{r}}{\left(\theta_{i}-e_{r}\right)}-\frac{c_{r}^{(1)}}{\left(\theta_{i}-e_{r}\right)^{2}}+\cdots+\frac{\left(p_{r}-1\right) c_{p_{r}-1}^{(r)}}{\left(\theta_{i}-e_{r}\right)^{p_{r}}}=0 .
\end{gathered}
$$

This is easy to see from the ansatz [Eq. (18)]. Indeed if we evaluate the differential equation using Eq. (18) at $x=\theta_{i}$, these equations are readily obtained.

Next we relate these finite solutions to the superintegrable system in $n$ dimensional Euclidean space from which they are obtained by separation of variables. To begin this process we first label Cartesian-type coordinates by $y_{j}^{J}, j=1, \ldots, p_{J}, J=0, \ldots, r$. In terms of the separable coordinates $x=u_{1}, \ldots, u_{n}$ the Cartesian-type coordinates are specified by the relations ${ }^{20,25}$

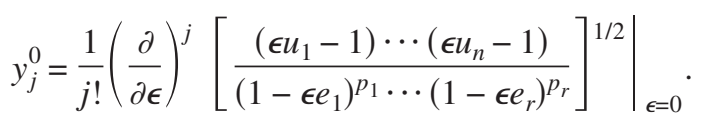

For coordinates corresponding to $y_{k}^{K}, k=1, \ldots, p_{K}$, and $K \neq 0$, we choose

$$
y_{k}^{K}=\frac{1}{(k-1) !}\left(\frac{\partial}{\partial e_{K}}\right)^{k-1}\left[\frac{\left(u_{1}-e_{K}\right) \cdots\left(u_{n}-e_{K}\right)}{\Pi_{J \neq K}\left(e_{K}-e_{J}\right)^{p_{J}}}\right]^{1 / 2} .
$$

These coordinates can be identified with the standard Cartesian coordinates via the quadratic form

$$
\sum_{s+t=p_{0}+1} y_{s}^{0} y_{t}^{0}+\sum_{J=1}^{r} \sum_{s+t=p_{J}} y_{s}^{J} y_{t}^{J}=x_{1}^{2}+\cdots+x_{n}^{2} .
$$

Indeed if we label the Cartesian coordinates by $x_{j}^{J}, j=1, \ldots, p_{J}, J=0, \ldots, r$, then we can make the choices

$$
y_{j}^{J}=\frac{1}{\sqrt{2}}\left(x_{j}^{J}+i x_{2 m+1-j}^{J}\right), \quad y_{j+m}^{J}=\frac{1}{\sqrt{2}}\left(x_{j}^{J}-i x_{2 m+1-j}^{J}\right),
$$

where $J=2 m, j=1, \ldots, m$, and 


$$
y_{j}^{J}=\frac{1}{\sqrt{2}}\left(x_{j}^{J}+i x_{2 m+2-j}^{J}\right), \quad y_{j+m+1}^{J}=\frac{1}{\sqrt{2}}\left(x_{j}^{J}-i x_{2 m+2-j}^{J}\right), \quad y_{m}^{J}=x_{m+1}^{J},
$$

where $J=2 m+1, j=1, \ldots m$. The infinitesimal distance is given by

$$
\mathrm{d} s^{2}=\sum_{I=0}^{r} \sum_{i=1}^{p_{I}}\left(\mathrm{~d} x_{i}^{I}\right)^{2}=\sum_{i=1}^{n} \frac{\Pi_{j \neq i}\left(u_{i}-u_{j}\right)}{P\left(u_{i}\right)} \mathrm{d} u_{i}^{2} .
$$

If we take Eq. (17) and allow $x$ to have the values $u_{1}, \ldots, u_{n}$ and $F=f\left(u_{1}, \ldots, u_{n}\right)$, then we can calculate each of the expressions $\hat{a}_{j}, j=n-1, \ldots, 0$, in terms of the general coordinates $y_{i}^{I}$. In permitting $x$ to take on these values we are identifying the resulting ordinary differential equations as the separation equations of a separable Schrödinger equation with potential in $n$ dimensions. To do this calculation we need to make use of the symmetric functions $S_{j}, j=1, \ldots, n$, defined by the expressions

$$
\begin{gathered}
S_{0}^{i}=1, \quad S_{1}^{i}=S_{1}^{i}\left[u_{1}, \ldots, u_{i-1}, u_{i+1}, \ldots, u_{n}\right]=\sum_{j=1}^{n} u_{j}, \quad j \neq i, \\
S_{2}^{i}=S_{2}^{i}\left[u_{1}, \ldots, u_{i-1}, u_{i+1}, \ldots, u_{n}\right]=\sum_{j \neq k}^{n} u_{k} u_{j}+\cdots, \quad k, j \neq i, \ldots, \\
S_{n-1}^{i}=S_{n-1}^{i}\left[u_{1}, \ldots, u_{i-1}, u_{i+1}, \ldots, u_{n}\right]=\prod_{j=1}^{n} u_{j}, \quad j \neq i .
\end{gathered}
$$

Previously we considered $\hat{a}_{j}, j=0, \ldots, n-1$, as an eigenvalue. Now we consider it as the symmetry operator with this eigenvalue. Solving the $n$ equations of type (17), we find

$$
\hat{a}_{j}=\sum_{i=1}^{n} \frac{1}{\prod_{k \neq i}\left(u_{i}-u_{k}\right)} S_{n-1-j}^{i} P_{i}
$$

where

$$
\begin{aligned}
P_{i}= & \sqrt{P\left(u_{i}\right)} \frac{\mathrm{d}}{\mathrm{d} u_{i}}\left(\sqrt{P\left(u_{i}\right)} \frac{\mathrm{d}}{\mathrm{d} u_{i}}\right)+a_{n+p_{0}} u_{i}^{n+p_{0}}+\cdots+a_{n} u_{i}^{n}+\frac{b_{1}^{(1)}}{\left(u_{i}-e_{1}\right)}+\frac{b_{2}^{(1)}}{\left(u_{i}-e_{1}\right)^{2}}+\cdots+\frac{b_{p}^{(1)}}{\left(u_{i}-e_{1}\right)^{p_{1}}} \\
& +\cdots+\frac{b_{1}^{(r)}}{\left(u_{i}-e_{r}\right)}+\frac{b_{2}^{(r)}}{\left(u_{i}-e_{r}\right)^{2}}+\cdots+\frac{b_{p}^{(r)}}{\left(u_{i}-e_{r}\right)^{p_{r}}} .
\end{aligned}
$$

If we look for an eigenfunction $F$ of the form

$$
F=\prod_{j=1}^{n} f\left(u_{j}\right) \Pi_{r=1}^{s}\left(u_{j}-\theta_{r}\right),
$$

then the expression for the second part of this, viz., $\Pi_{j=1}^{n} \Pi_{r=1}^{s}\left(u_{j}-\theta_{r}\right)$, can be written in terms of the $y_{i}^{I}$ via the identity

$$
S_{0}^{0} \lambda^{p_{0}-1}+S_{1}^{0} \lambda^{p_{0}-2}+\cdots+S_{p_{0}-1}^{0}+\sum_{J=1}^{r} \sum_{j=1}^{p_{J}} \frac{S_{p_{J}+2-j}^{J}}{\left(\lambda-e_{J}\right)^{j}}=\frac{\Pi_{j=1}^{n}\left(u_{j}-\lambda\right)}{P(\lambda)},
$$

where $S_{j}^{J}=\Sigma_{\ell+k=s} y_{\ell}^{J} y_{k}^{J}$.

The product function $\Pi_{j=1}^{n} f\left(u_{j}\right)$ can be written in terms of the $y_{i}^{I}$ variables via our implicit evaluation of symmetric functions $S_{j}\left[u_{1}, \ldots, u_{n}\right]$ in terms of the $y_{i}^{I}$. We can determine that $\hat{a}_{n-1}$ has the form of an $n$ dimensional Laplacian plus a potential which is rational in the coordinates $y_{i}^{I}$. 
We now take a closer look at $H=\hat{a}_{n-1}$. The differential operator part of this term can be identified with the Laplacian of the operator associated with the metric $\mathrm{d} s^{2}$. The various parts of the potential can be associated with the calculation of Stäckel multiplier terms of the form

$$
\sum_{i=1}^{n} \frac{1}{\Pi_{k \neq i}\left(u_{i}-u_{k}\right)} Q_{i}
$$

where $Q_{i}=u_{i}^{s}$ or $Q_{i}\left(u_{i}-e_{J}\right)^{-t}$, corresponding to the coefficients $a_{s}$ or $b_{t}^{(J)}$ of Eq. (17). We can calculate a basis for these Stäckel multipliers in the $y_{i}^{I}$ coordinates. For Stäckel multipliers with terms of the form $Q_{i}=u_{i}^{s}$, we observe the identity

$$
\frac{\Pi_{I=1}^{r}\left(1-\epsilon e_{I}\right)}{\Pi_{i=1}^{n}\left(\epsilon u_{i}-1\right)}=\sum_{i=1}^{n} \frac{u_{i}^{p_{0}-1} \Pi_{J=1}^{r}\left(u_{i}-e_{J}\right)^{p_{J}}}{\Pi_{j \neq i}\left(u_{j}-u_{i}\right)\left(\epsilon u_{i}-1\right)} .
$$

From this identity we can calculate combinations of Stäckel multipliers of the form with $Q=u_{i}^{s}$ and $s>n-1$. Indeed we differentiate according to

$$
\left.\left(\frac{\partial}{\partial \epsilon}\right)^{t}\left[\frac{\Pi_{I=1}^{r}\left(1-\epsilon e_{I}\right)}{\Pi_{i=1}^{n}\left(\epsilon u_{i}-1\right)}\right]\right|_{\epsilon=0}=\left.\sum_{i=1}^{n}(-1)^{t} t ! \frac{u_{i}^{p_{0}+t-1} \Pi_{J=1}^{r}\left(u_{i}-e_{J}\right)^{p_{J}}}{\prod_{j \neq i}\left(u_{j}-u_{i}\right)\left(\epsilon u_{i}-1\right)}\right|_{\epsilon=0},
$$

where $1 \leqslant t \leqslant p_{0}+1$.

From this formula we see that we can we can construct Stäckel multipliers in which the $Q_{i}$ are polynomials in $u_{i}$, where the maximal order is $n+t-1$. Further, we can explicitly calculate these in terms of the coordinates $y_{i}^{0}$. This can be seen from the formulas for $S_{s}^{0}$. If we define

$$
\frac{1}{\left(\tilde{y}_{0}^{0}\right)^{2}}=\frac{\Pi_{I=1}^{r}\left(1-\epsilon e_{I}\right)}{\Pi_{i=1}^{n}\left(\epsilon u_{i}-1\right)}=\sum_{i=1}^{n} \frac{u_{i}^{p_{0}-1} \Pi_{J=1}^{r}\left(u_{i}-e_{J}\right)^{p_{J}}}{\Pi_{j \neq i}\left(u_{j}-u_{i}\right)\left(\epsilon u_{i}-1\right)},
$$

then the terms that we need for our Stäckel multipliers are

$$
\tilde{Y}_{t}^{0}=\left.\left(\frac{\partial}{\partial \epsilon}\right)^{t}\left[\frac{1}{\left(\tilde{y}_{0}^{0}\right)^{2}}\right]\right|_{\epsilon=0}, \quad t=1, \ldots, p_{0}+1,
$$

where we can use the recurrence formula $\partial / \partial \epsilon\left(\widetilde{y}_{j}^{0}\right)=(j+1) \widetilde{y}_{j+1}^{0}$ and, once all the calculations are done, we put $\epsilon \rightarrow 0$ and then $\widetilde{y}_{j}^{0} \rightarrow y_{j}^{0}$.

In this way we can construct basis elements for those parts of our potential which are entirely polynomial in the $u_{i}$ variables. For the parts that are singular in these variables we use the identity

$$
\frac{\prod_{J \neq K}\left(e_{K}-e_{J}\right)^{p_{J}}}{\prod_{i=1}^{n}\left(u_{i}-e_{K}\right)}=\sum_{i=1}^{n} \frac{\Pi_{J \neq K}\left(u_{i}-e_{J}\right)^{p_{J}}}{\left(u_{i}-e_{K}\right) \Pi_{j \neq i}\left(u_{i}-u_{j}\right)} .
$$

The right hand side of this expression is in the form of a Stäckel multiplier with $Q_{i}=\prod_{J \neq K}\left(u_{i}\right.$ $\left.-e_{J}\right)^{p} /\left(u_{i}-e_{K}\right)$, which can be written as a polynomial in $u_{i}$ plus a term $1 /\left(u_{i}-e_{K}\right)$. We see immediately that Stäckel multipliers containing terms of the form $1 /\left(u_{i}-e_{k}\right)^{t}$, where $t \geqslant 2$, can be generated by differentiating this identity $t-1$ times with respect to $e_{K}$. Indeed we recognize that

$$
\frac{\prod_{J \neq K}\left(e_{K}-e_{J}\right)^{p_{J}}}{\prod_{i=1}^{n}\left(u_{i}-e_{K}\right)}=\frac{1}{\left(y_{1}^{K}\right)^{2}} .
$$

The additional terms that arise in the potential and complete our basis have the form

$$
Y_{t}^{K}=\left(\frac{\partial}{\partial e_{k}}\right)^{t}\left[\frac{1}{\left(y_{1}^{K}\right)^{2}}\right], \quad t=0, \ldots, p_{K}-1 .
$$

These expressions can be determined in terms of the $y_{s}^{K}$ via the recursion relation 


$$
\frac{\partial}{\partial e_{K}}\left(y_{k}^{K}\right)=(k+1) y_{k+1}^{K} .
$$

Indeed we could establish the eigenvalues of the operators $\hat{a}_{j}$ by acting on the functions $\Phi\left(u_{1}, \ldots, u_{n}\right)$ when expressed in terms of a basis of symmetric polynomials in the variables $u_{i}$. In general these expressions are of a different form than those obtained from the single separated equation and are easier to calculate.

This procedure is best illustrated by a specific example. If we choose $P(x)=(x-1)^{2} x$ and $n$ $=4$, then the corresponding separated equation is

$$
\left\{\sqrt{P(\lambda)} \frac{\mathrm{d}}{\mathrm{d} \lambda}\left(\sqrt{P(\lambda)} \frac{\mathrm{d}}{\mathrm{d} \lambda}+\left(a_{5} \lambda^{5}+a_{4} \lambda^{4}+\hat{a}_{3} \lambda^{3}+\hat{a}_{2} \lambda^{2}+\hat{a}_{1} \lambda+\hat{a}_{0}+\frac{b_{1}}{\lambda-1}+\frac{b_{2}}{(\lambda-1)^{2}}+\frac{c_{1}}{\lambda}\right)\right\} f(\lambda)=0 .\right.
$$

If we consider this equation in isolation then we can find polynomial solutions as follows. Making the assignments

$$
a_{5}=-4 \ell_{4}^{2}, \quad a_{4}=4 \ell_{4}\left(2 \ell_{4}-\ell_{5}\right), \quad b_{1}=\ell_{6}\left(-\ell_{6}+2 p-1\right), \quad b_{2}=-\ell_{6}^{2}, \quad c_{1}=-\frac{1}{2} q(2 q-1),
$$

we can look for solutions of the form

$$
f(\lambda)=\exp \left(\ell_{4} \lambda^{2}+\ell_{5} \lambda+\frac{\ell_{6}}{\lambda-1}\right)(\lambda-1)^{p} \lambda^{q} \Pi_{r=1}^{s}\left(\lambda-\theta_{r}\right),
$$

where the $\theta_{j}$ satisfy the relation

$$
4 \ell_{5}+8 \ell_{4} \theta_{i}+\frac{2(2 p+1)}{\theta_{i}-1}-\frac{6 \ell_{6}}{\left(\theta_{i}-1\right)^{2}}+\frac{4 q+1}{\theta_{i}}-\sum_{j \neq i} \frac{4}{\theta_{i}-\theta_{j}}=0 .
$$

The values of the constants $\hat{a}_{j}, j=0, \ldots, 3$, can be represented in terms of the $\theta_{i}$ via the formulas

$$
\begin{gathered}
\hat{a}_{3}=-\ell_{5}^{2}-4 \ell_{4}^{2}+8 \ell_{4} \ell_{5}-[4(p+q+s)+5] \ell_{4}, \\
\hat{a}_{2}=-2 \ell_{5}^{2}-4 \ell_{4} \ell_{5}+4 \ell_{4} \ell_{6}-\left[2(p+q+s)+\frac{3}{2}\right] \ell_{5}+4(p+2 q+2 s+2) \ell_{4}-4 \ell_{4} \sum_{i=1}^{s} \theta_{i}, \\
\hat{a}_{1}=-\ell_{5}^{2}+2 \ell_{5} \ell_{6}+2[p+1+2 q+2 s] \ell_{5}-[4 s+4 q+3] \ell_{4}-(p+q)\left(p+q+\frac{1}{2}\right)-s\left(s+\frac{1}{2}\right) \\
-2(p+q) s+\left(-2 \ell_{5}+8 \ell_{4}\right) \sum_{i=1}^{s} \theta_{i}-4 \ell_{4} \sum_{i=1}^{s} \theta_{i}^{2}, \\
\hat{a}_{0}=-\left(2 q+2 s+\frac{1}{2}\right) \ell_{5}+\left(2 p+2 q+2 s-\frac{1}{2}\right) \ell_{6}+\left[4 \ell_{5}-4 \ell_{4}-2 p-2 q-\frac{1}{2}(4 s-1)\right] \\
\times \sum_{i=1}^{s} \theta_{i}+\left(-2 \ell_{5}+8 \ell_{4}\right) \sum_{i=1}^{s} \theta_{i}^{2}-4 \ell_{4} \sum_{i=1}^{s} \theta_{i}^{3} .
\end{gathered}
$$

We now show the superintegrable context of these observations. Consider the coordinates in four dimensions given by the formulas

$$
y_{1}^{2}=-4(s-1)(t-1)(u-1)(v-1), \quad y_{4}=-2(s+t+u+v-2),
$$




$$
2 y_{1} y_{2}=4(3-2(s+t+u+v)+(s t+s u+s v+t u+t v+u v)-s t u v), \quad y_{3}^{2}=-4 s t u v,
$$

where we identify four dimensional Cartesian coordinates as

$$
y_{1}=\frac{1}{\sqrt{2}}(x+i y), \quad y_{2}=\frac{1}{\sqrt{2}}(x-i y), \quad y_{3}=z, \quad y_{4}=t .
$$

The appropriate superintegrable system in four dimensions is

$$
H=\Delta+\frac{\alpha}{y_{1}^{2}}+\frac{\beta y_{2}}{y_{1}^{3}}+\frac{\gamma}{y_{3}^{2}}+\delta\left(4 y_{4}^{2}+2 y_{1} y_{2}+y_{3}^{2}\right)+\mu y_{4},
$$

where $\Delta=\partial_{x}^{2}+\partial_{y}^{2}+\partial_{z}^{2}+\partial_{t}^{2}$. In these coordinates the separation equations for $H \Psi=E \Psi$ have exactly the form (20) with the identifications

$$
\alpha=i 4(1-2 p), \quad \beta=-8 \ell_{6}^{2}, \quad \gamma=-2 q(2 q-1), \quad \delta=-\ell_{4}^{2}, \quad \mu=4 \ell_{4} \ell_{5},
$$

and identification of $\hat{a}_{3}$ with $E$. Indeed, if we use coordinates $y_{1}, y_{2}, y_{3}$, and $y_{4}$ in the Schrödinger equation $\Delta \Psi=E \Psi$ and employ the relationship

$$
\frac{2 y_{1} y_{2}}{L-1}+\frac{y_{1}^{2}}{(L-1)^{2}}+\frac{y_{3}^{2}}{L}-2 y_{4}-L=-\frac{(u-L)(v-L)(s-L)(t-L)}{L(L-1)^{2}},
$$

we can find finite solutions of the form

$$
\begin{aligned}
\Psi= & \exp \left(\frac{1}{2} \ell_{4}\left(2 y_{1} y_{2}+y_{3}^{2}+2 y_{4}^{2}\right)-\ell_{5} y_{4}+2 i \ell_{6} \frac{y_{2}}{y_{1}}\right) y_{1}^{p / 2} y_{3}^{q / 2} \\
& \times \Pi_{r=1}^{s}\left(\frac{2 y_{1} y_{2}}{\theta_{r}-1}+\frac{y_{1}^{2}}{\left(\theta_{r}-1\right)^{2}}+\frac{y_{3}^{2}}{\theta_{r}}-2 y_{4}-\theta_{r}\right),
\end{aligned}
$$

where the same equations for the zeros are satisfied. This method of derivation is in complete analogy with that used by Whittaker and Watson. ${ }^{21}$ The $\hat{a}_{2}, \hat{a}_{1}, \hat{a}_{0}$ are then separation constants. A useful feature of this observation is the availability of direct algebraic methods to determine the eigenvalues of these parameters. Indeed in this case if we write (in operator form)

$$
\begin{aligned}
\hat{a}_{i}= & \frac{1}{(s-t)(s-u)(s-v)} P_{s}^{i}+\frac{1}{(t-u)(t-s)(t-v)} P_{t}^{i}+\frac{1}{(u-t)(u-s)(u-v)} P_{u}^{i} \\
& +\frac{1}{(v-t)(v-u)(v-s)} P_{v}^{i},
\end{aligned}
$$

then typically

$$
\begin{gathered}
P_{s}^{3}=\sqrt{P(s)} \frac{\partial}{\partial s}\left(\sqrt{P(s)} \frac{\partial}{\partial s}\right)+\left(a_{1} s^{5}+a_{2} s^{4}+\frac{b_{1}}{s-1}+\frac{b_{2}}{(s-1)^{2}}+\frac{c_{1}}{s}\right), \\
P_{s}^{4}=(t+u+v) P_{s}^{3}, \quad P_{s}^{5}=(t u+t v+u v) P_{s}^{3}, \quad P_{s}^{6}=t u v P_{s}^{3} .
\end{gathered}
$$

Since the products of separated eigenfunctions are symmetric in $s, t, u, v$, we see that eigenvalues of $\hat{a}_{i}$ can be obtained by acting on a basis of symmetric functions. We consider the symmetric functions

$$
S_{0}=1, \quad S_{1}=s+t+u+v, \quad S_{2}=s t+s u+s v+t u+t v+u v,
$$




$$
S_{3}=s t u+t u v+s u v+s t v, \quad S_{4}=s t u v .
$$

We can, as a particular example, look for solutions of the form

$$
\begin{aligned}
\Psi= & \exp \left(\ell_{4}\left(s^{2}+t^{2}+u^{2}+v^{2}\right)+\ell_{5}(s+t+u+v)+\ell_{6}\left(\frac{1}{s-1}+\frac{1}{t-1}+\frac{1}{u-1}+\frac{1}{v-1}\right)[(s-1)(t-1)\right. \\
& \times(u-1)(v-1)]^{p}(s t u v)^{q}\left[B_{0} S_{0}+B_{1} S_{1}+B_{2} S_{2}+B_{3} S_{3}\right] .
\end{aligned}
$$

If we look for eigenvalues of $\hat{a}_{i}, i=0, \ldots, 3$, we obtain the determinantal equations for the corresponding eigenvalues $a_{i}$. In the simplified case for which $\ell_{4}=\ell_{5}=\ell_{6}=p=q=1$, we find

$$
\begin{gathered}
\left(a_{3}-18\right)\left(a_{3}-14\right) 4=0, \quad\left(2 a_{2}-33\right)\left(4 a_{2}^{4}-1344 a_{2}^{3}+42472 a_{2}^{2}-596992 a_{2}+3141805\right)=0 \\
\left(a_{1}-3\right)\left(4 a_{1}^{4}-92 a_{1}^{3}+1005 a_{1}^{2}-6318 a_{1}+15641\right)=\left(2 a_{0}-11\right)\left(4 a_{0}^{4}-120 a_{0}^{3}+1309 a_{0}^{2}-6321 a_{0}\right. \\
+11723)=0 .
\end{gathered}
$$

\section{POLYNOMIAL SOLUTIONS FROM SUPERINTEGRABLE SYSTEMS ON THE COMPLEX $n$ SPHERE}

Applications of superintegrable systems on the complex $n$-sphere proceed in analogy with the approach in complex Euclidean $n$-space. There is an important subclass of such systems with nondegenerate potentials that can be constructed for all $n \geqslant 2$, based on their relationship to variable separation in generic Jacobi elliptic coordinates. The prototype superintegrable system which is nondegenerate on the $n$ dimensional sphere has the Hamiltonian

$$
H=\sum_{i=0}^{n}\left(\partial_{i}^{2}+\frac{\beta_{i}}{s_{i}^{2}}\right)+\delta, \quad \partial_{i}=\partial_{s_{i}}
$$

where $\sum_{i=0}^{n} s_{i}^{2}=1$. The system $H \Psi=E \Psi$ has a basis of $n(n+1) / 2$ second order symmetry operators given by

$$
M_{i j}=M_{j i}=\left(s_{i} \partial_{j}-s_{j} \partial_{i}\right)^{2}+\beta_{i} \frac{s_{j}^{2}}{s_{i}^{2}}+\beta_{j} \frac{s_{i}^{2}}{s_{j}^{2}}, \quad i \neq j .
$$

Again, all are dependent on a subset of $2 n-1$ functionally independent symmetries. The corresponding equation $H \Psi=E \Psi$ admits multiplicative separation in the generic $n$ dimensional elliptical coordinates.

$$
s_{i}^{2}=\Pi_{j=1}^{n}\left(u_{j}-e_{i}\right) / \Pi_{k \neq i}\left(e_{k}-e_{i}\right)
$$

simultaneously for all values of the parameters with $e_{i} \neq e_{k}$ if $i \neq k$ and $i, k=0, \ldots, n$. If we were dealing with the real sphere then we could assume that the inequalities $e_{1}<e_{2}<\cdots<e_{n}$ and $e_{0}$ $<u_{1}<e_{1}<\ldots<e_{n-1}<u_{n}<e_{n}$ hold. Thus the equation is multiseparable and separates in a continuum of elliptic coordinate systems (and in others besides).

The infinitesimal distance in Jacobi elliptical coordinates $u_{j}$ has the form

$$
\mathrm{d} s^{2}=\sum_{i=1}^{n} \frac{\Pi_{j \neq i}\left(u_{i}-u_{j}\right)}{P\left(u_{i}\right)} \mathrm{d} u_{i}^{2}
$$

where $P(\lambda)=-4 \Pi_{k=0}^{n}\left(\lambda-e_{k}\right)$. Here $e_{j} \neq e_{k}$ for $j \neq k$. However, it is well known that Eq. (22) is a metric on the sphere for any polynomial $P(\lambda)$ of order $n+1$ and that each choice of such a $P(\lambda)$ defines an elliptic-type multiplicative separable solution of the Laplace-Beltrami eigenvalue problem (with constant potential) on the complex $n$-sphere. The distinct cases are labeled by the multiplicities of the $n+1$ roots. If for each distinct case we determine the most general potential 
that admits separation for all $e_{j}$ compatible with the multiplicity structure of the roots, we determine a unique superintegrable system with nondegenerate potential and $n(n+1) / 2$ second order symmetries. ${ }^{10,25}$ These are the generic superintegrable systems on the sphere. [Thus, for $n=3$ there are five distinct cases for $-\frac{1}{4} P(\lambda)$ :

$$
\begin{gathered}
\left(\lambda-e_{0}\right)\left(\lambda-e_{1}\right)\left(\lambda-e_{2}\right)\left(\lambda-e_{3}\right), \quad\left(\lambda-e_{0}\right)\left(\lambda-e_{1}\right)\left(\lambda-e_{2}\right)^{2}, \quad\left(\lambda-e_{0}\right)^{2}\left(\lambda-e_{1}\right)^{2}, \\
\left(\lambda-e_{0}\right)\left(\lambda-e_{1}\right)^{3}, \quad\left(\lambda-e_{0}\right)^{4},
\end{gathered}
$$

where $e_{j} \neq e_{k}$ for $j \neq k$. The first case corresponds to Jacobi elliptic coordinates.] The number of distinct generic superintegrable systems for each integer $n \geqslant 2$ is $p(n+1)$, where $p(j)$ is the number of integer partitions of $j$.

As in the Euclidean case, all of the generic separable systems, their potentials, and their defining symmetries can be obtained from the basic Jacobi elliptic system in $n$ dimensions by a complicated but well defined set of limit processes. ${ }^{25}$ Although we cannot write down master canonical expressions for all such generic systems in Cartesian-like coordinates $s_{k}$, it is easy to take these limits and write down a master equation for the separated ordinary differential equations in the elliptic coordinates, $u_{i}$. The separation equation for each of the coordinates $x=u_{i}$ is essentially of the same form,

$$
\begin{aligned}
& \sqrt{P(x)} \frac{\mathrm{d}}{\mathrm{d} x}\left(\sqrt{P(x)} \frac{\mathrm{d} F}{\mathrm{~d} x}\right)+\left(a_{n-1} x^{n-1}+\cdots+\hat{a}_{0} \frac{b_{1}^{(1)}}{\left(x-e_{1}\right)}+\frac{b_{2}^{(1)}}{\left(x-e_{1}\right)^{2}}+\cdots+\frac{b_{p_{1}}^{(1)}}{\left(x-e_{1}\right)^{p_{1}}}\right. \\
& \left.+\cdots+\frac{b_{1}^{(r)}}{\left(x-e_{r}\right)}+\frac{b_{2}^{(r)}}{\left(x-e_{r}\right)^{2}}+\cdots+\frac{b_{p_{r}}^{(r)}}{\left(x-e_{r}\right)^{p_{r}}}\right) F=0,
\end{aligned}
$$

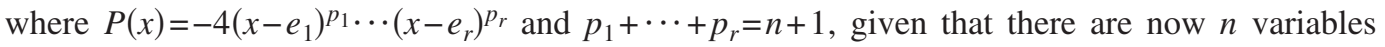
$u_{1}, \ldots, u_{n}$. Much of what has already been done for Euclidean space coordinates carries over, with the essential difference that there is no singularity at infinity. If we look for finite solutions we can proceed as before. We seek solutions of the form

$$
\begin{aligned}
& \Psi=\exp \left(\frac{c_{1}^{(1)}}{\left(x-e_{1}\right)}+\frac{c_{2}^{(1)}}{\left(x-e_{1}\right)^{2}}+\cdots+\frac{c_{p_{1}-1}^{(1)}}{\left(x-e_{p_{1}}\right)^{p_{1}-1}}+\cdots+\frac{c_{1}^{(r)}}{\left(x-e_{r}\right)}+\frac{c_{2}^{(r)}}{\left(x-e_{r}\right)^{2}}+\cdots+\frac{c_{p_{r}-1}^{(r)}}{\left(x-e_{r}\right)^{p_{r}-1}}\right)
\end{aligned}
$$

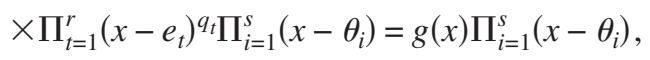

for which the zeros of the polynomials satisfy

$$
\begin{aligned}
0= & \sum_{j \neq i} \frac{1}{\theta_{i}-\theta_{j}}+\cdots+\frac{1 / 4 p_{1}+q_{1}}{\left(\theta_{i}-e_{1}\right)}-\frac{c_{1}^{(1)}}{\left(\theta_{i}-e_{1}\right)^{2}}+\cdots+\frac{\left(p_{1}-1\right) c_{p_{1}-1}}{\left(\theta_{i}-e_{1}\right)^{p_{1}}} \cdots+\frac{1 / 4 p_{r}+q_{r}}{\left(\theta_{i}-e_{r}\right)}-\frac{c_{r}^{(1)}}{\left(\theta_{i}-e_{r}\right)^{2}} \\
& +\cdots+\frac{\left(p_{r}-1\right) c_{p_{r}-1}}{\left(\theta_{i}-e_{r}\right)^{p_{r}}} .
\end{aligned}
$$

To relate these finite solutions to superintegrable systems on the sphere, we introduce a set of coordinates that we again call $y_{k}^{K}, k=1, \ldots, p_{k}, K=1, \ldots, r$, and which are given by the formula

$$
y_{k}^{K}=\frac{1}{(k-1) !}\left(\frac{\partial}{\partial e_{K}}\right)^{k-1}\left[\frac{\left(u_{1}-e_{K}\right) \cdots\left(u_{n}-e_{K}\right)}{\Pi_{J \neq K}\left(e_{K}-e_{J}\right)^{p_{J}}}\right]^{1 / 2} .
$$

These coordinates can be identified with the usual coordinates on a sphere via the relation 


$$
\sum_{K=1}^{r} \sum_{s+t=p_{K}} y_{s}^{K} y_{t}^{K}=1
$$

This is done via the assignment

$$
\begin{gathered}
y_{j}^{J}=\frac{1}{\sqrt{2}}\left(s_{j}^{J}+i s_{2 m+1-j}^{J}\right), \quad y_{j+m}^{J}=\frac{1}{\sqrt{2}}\left(s_{j}^{J}-i s_{2 m+1-j}^{J}\right), \quad J=2 m, \quad j=1, \ldots, m, \\
y_{j}^{J}=\frac{1}{\sqrt{2}}\left(s_{j}^{J}+i s_{2 m+2-j}^{J}\right), \quad y_{j+m+1}^{J}=\frac{1}{\sqrt{2}}\left(s_{j}^{J}-i s_{2 m+2-j}^{J}\right), \quad y_{m}^{J}=s_{m+1}^{J},
\end{gathered}
$$

where

$$
\sum_{K=1}^{r} \sum_{k=1}^{p_{k}}\left(s_{k}^{K}\right)^{2}=1
$$

The infinitesimal distance is given by

$$
\mathrm{d} s^{2}=\sum_{K=1}^{r} \sum_{k=1}^{p_{K}}\left(\mathrm{~d} s_{k}^{K}\right)^{2}=\sum_{i=1}^{n} \frac{\Pi_{j \neq i}\left(u_{i}-u_{j}\right)}{P\left(u_{i}\right)} \mathrm{d} u_{i}^{2} .
$$

Solving the $n$ equations of type (23), we find the operator expressions

$$
\hat{a}_{j}=\sum_{i=1}^{n} \frac{1}{\prod_{k \neq i}\left(u_{i}-u_{k}\right)} S_{n-1-j}^{i} P_{i},
$$

where

$$
\begin{aligned}
P_{i}= & \sqrt{P\left(u_{i}\right)} \frac{\mathrm{d}}{\mathrm{d} u_{i}}\left(\sqrt{P\left(u_{i}\right)} \frac{\mathrm{d}}{\mathrm{d} u_{i}}\right)+\left(\frac{b_{1}^{(1)}}{\left(u_{i}-e_{1}\right)}+\frac{b_{2}^{(1)}}{\left(u_{i}-e_{1}\right)^{2}}+\cdots+\frac{b_{p}^{(1)}}{\left(u_{i}-e_{1}\right)^{p_{1}}}+\cdots+\frac{b_{1}^{(r)}}{\left(u_{i}-e_{r}\right)}\right. \\
& \left.+\frac{b_{2}^{(r)}}{\left(u_{i}-e_{r}\right)^{2}}+\cdots+\frac{b_{p}^{(r)}}{\left(u_{i}-e_{r}\right)^{p_{r}}}\right) .
\end{aligned}
$$

We look for an eigenfunction of the form

$$
F=\Pi_{j=1}^{n} g\left(u_{j}\right) \Pi_{r=1}^{s}\left(u_{j}-\theta_{r}\right) .
$$

Then the second part of this expression, viz., $\Pi_{j=1}^{n} \Pi_{r=1}^{s}\left(u_{j}-\theta_{r}\right)$, can be written in terms of the $y_{i}^{I}$ via the identity

$$
\sum_{J=1}^{r} \sum_{j=1}^{p_{J}} \frac{S_{p_{J}+2-j}^{J}}{\left(\lambda-e_{J}\right)^{j}}=\frac{\Pi_{j=1}^{n}\left(u_{j}-\lambda\right)}{P(\lambda)} .
$$

The product function $\Pi_{j=1}^{n} g\left(u_{j}\right)$ can be written in terms of the $y_{i}^{I}$ variables via our implicit evaluation of symmetric functions $S_{j}\left[u_{1}, \ldots, u_{n}\right]$ in terms of the $y_{i}^{I}$. We can determine that $\hat{a}_{n-1}$ has the form of an $n$ dimensional Laplacian plus a potential which is rational in the coordinates $y_{i}^{I}$. If we look at $H=\hat{a}_{n-1}$, we can identify the differential operator part with the Laplacian of the operator associated with the metric $\mathrm{d} s^{2}$. The various parts associated with the potential require the calculation of the Stäckel multiplier terms of the form 


$$
\sum_{i=1}^{n} \frac{1}{\Pi_{k \neq i}\left(u_{i}-u_{k}\right)} Q_{i}
$$

where $Q_{i}=\left(u_{i}-e_{J}\right)^{-t}$ corresponding to the coefficients $b_{t}^{(J)}$ of Eq. (23). We can readily calculate a basis for these Stäckel multipliers in terms of the $y_{i}^{I}$ coordinates. For the parts that are singular in these variables we proceed as previously. We first observe the identity

$$
\frac{\Pi_{J \neq K}\left(e_{K}-e_{J}\right)^{p_{J}}}{\Pi_{i=1}^{n}\left(u_{i}-e_{K}\right)}=\sum_{i=1}^{n} \frac{\Pi_{J \neq K}\left(u_{i}-e_{J}\right)^{p_{J}}}{\left(u_{i}-e_{K}\right) \Pi_{j \neq i}\left(u_{i}-u_{j}\right)} .
$$

Note that the right hand side of this expression is in the form of a Stäckel multiplier with $Q_{i}$ $=\Pi_{J \neq K}\left(u_{i}-e_{J}\right)^{p} /\left(u_{i}-e_{K}\right)$, which can be written as a polynomial in $u_{i}$ plus a term $1 /\left(u_{i}-e_{K}\right)$. We see immediately that Stäckel multipliers containing terms of the form $1 /\left(u_{i}-e_{k}\right)^{t}$, where $t \geqslant 2$, can be generated by differentiating this identity $t-1$ times with respect to $e_{K}$. Indeed,

$$
\frac{\Pi_{J \neq K}\left(e_{K}-e_{J}\right)^{p_{J}}}{\prod_{i=1}^{n}\left(u_{i}-e_{K}\right)}=\frac{1}{\left(y_{1}^{K}\right)^{2}} .
$$

The additional terms that arise in the potential, and complete our basis, have the form

$$
Y_{t}^{K}=\left(\frac{\partial}{\partial e_{k}}\right)^{t}\left[\frac{1}{\left(y_{1}^{K}\right)^{2}}\right], \quad t=0, \ldots, p_{K}-1 .
$$

These expressions can be determined in terms of the $y_{s}^{K}$ via the recursion relation

$$
\frac{\partial}{\partial e_{K}}\left(y_{k}^{K}\right)=(k+1) y_{k+1}^{K} \text {. }
$$

We could now establish the eigenvalues of the operators $\hat{a}_{j}$ by acting on the functions $\Phi\left(u_{1}, \ldots, u_{n}\right)$ expressed in terms of a basis of symmetric polynomials in the variables $u_{i}$. The observations we have made are best illustrated by a specific example. If we choose $P(x)=(x$ $-1)^{2} x$ and $n=2$, then the ordinary differential equation is

$$
\left\{\sqrt{P(\lambda)} \frac{\mathrm{d}}{\mathrm{d} \lambda}\left(\sqrt{P(\lambda)} \frac{\mathrm{d}}{\mathrm{d} \lambda}+\left(\hat{a}_{1} \lambda+\hat{a}_{0}+\frac{b_{1}}{\lambda-1}+\frac{b_{2}}{(\lambda-1)^{2}}+\frac{c_{1}}{\lambda}\right)\right)\right\} f(\lambda)=0 .
$$

If we consider this equation in isolation, then we can find polynomial solutions as follows. We make the assignments

$$
c_{1}=-p\left(p+\frac{1}{2}\right), \quad b_{1}=-\ell(\ell-1)+2 \ell q, \quad b_{2}=-\ell^{2},
$$

and look for solutions of the form

$$
f(\lambda)=\exp \left(\frac{\ell}{\lambda-1}\right)(\lambda-1)^{q} \lambda^{p} \Pi_{r=1}^{s}\left(\lambda-\theta_{r}\right),
$$

where the $\theta_{j}$ satisfy the relation

$$
\frac{2(3+2 q)}{\theta_{i}-1}-\frac{\ell^{2}}{\left(\theta_{i}-1\right)^{2}}+\frac{3+4 q}{\theta_{i}}+\sum_{j \neq i} \frac{4}{\theta_{i}-\theta_{j}}=0 .
$$

The values of the constants $\hat{a}_{j}, j=0,1$, can be represented in terms of the $\theta_{i}$ via the formulas

$$
\hat{a}_{1}=-\frac{1}{2}(p+q+s+2)(2 p+2 q+2 s+3)
$$




$$
\begin{aligned}
\hat{a}_{0}= & 2 s(2 p+q+s+2)+\ell\left(2 p+2 q+2 s+\frac{5}{2}\right)+2 p(p+q)+4 p+\frac{3}{2} q+2 \\
& +\left\{-2(p+q)-\frac{1}{2}(5+4 s)\right\} \sum_{r=1}^{s} \theta_{r} .
\end{aligned}
$$

To display the superintegrable context of these observations we employ the coordinates on the 2-sphere given by the formulas

$$
s_{1}^{2}+s_{2}^{2}=1-u v, \quad\left(s_{1}+i s_{2}\right)^{2}=(u-1)(v-1), \quad s_{3}^{2}=u v
$$

and consider the superintegrable system ${ }^{10}$

$$
H=p_{s_{1}}^{2}+p_{s_{2}}^{2}+p_{s_{3}}^{2}+\alpha \frac{1}{\left(s_{1}+i s_{2}\right)^{2}}+\beta \frac{s_{1}-i s_{2}}{\left(s_{1}+i s_{2}\right)^{3}}+\frac{\gamma}{s_{3}^{2}} .
$$

In these coordinates the separation equations for $H \Psi=E \Psi$ have exactly the form (24) with

$$
\alpha=\ell(2 q-1), \quad \beta=-2 \ell^{2}, \quad \gamma=-p\left(p+\frac{1}{2}\right),
$$

and the identification of $\hat{a}_{1}$ with $E$. Indeed, using coordinates $y_{1}, y_{2}$, and $y_{3}$ in the Schrödinger equation $\Delta \Psi=E \Psi$ and the relationship

$$
\frac{2\left(s_{1}^{2}+s_{2}^{2}\right)}{L-1}+\frac{\left(s_{1}+i s_{2}\right)^{2}}{(L-1)^{2}}+\frac{s_{3}^{2}}{L}=\frac{(u-L)(v-L)}{L(L-1)^{2}},
$$

we can find finite solutions of the form

$$
\Psi=\exp \left[-2 \ell\left(\frac{s_{1}+i s_{2}}{s_{1}-i s_{2}}\right)\right] s_{3}^{p / 2}\left(s_{1}+i s_{2}\right)^{q / 2} \Pi_{r=1}^{s}\left(\frac{2\left(s_{1}^{2}+s_{2}^{2}\right)}{\theta_{r}-1}+\frac{\left(s_{1}+i s_{2}\right)^{2}}{\left(\theta_{r}-1\right)^{2}}+\frac{s_{3}^{2}}{\theta_{r}}\right),
$$

where the same equations for the zeros are satisfied. This method of derivation is in complete analogy with that used by Whittaker and Watson. The $\hat{a}_{1}, \hat{a}_{0}$ are separation constants. A useful feature of this observation is the availability of direct algebraic methods to determine the eigenvalues of these parameters. We write

$$
\hat{a}_{1}=\frac{1}{(u-v)}\left[P_{u}-P_{v}\right], \quad \hat{a}_{0}=\frac{1}{(u-v)}\left[v P_{u}-u P_{v}\right]
$$

where

$$
P_{\lambda}=\sqrt{P(\lambda)} \frac{\partial}{\partial \lambda}\left(\sqrt{P(\lambda)} \frac{\partial}{\partial \lambda}\right)+\left(a_{1} \lambda+a_{0}+\frac{b_{1}}{s-1}+\frac{b_{2}}{(s-1)^{2}}+\frac{c_{1}}{s}\right) .
$$

We can now demonstrate that eigenvalues of $\hat{a}_{i}$ can be obtained by acting on a basis of symmetric functions. As a particular example, if we look for solutions of the form

$$
\Psi=\exp \left(\frac{\ell}{u-1}+\frac{\ell}{v-1}\right)[(u-1)(v-1)]^{q}(u v)^{p}\left(k_{1} u v+k_{2}(u+v)+k_{3}\right),
$$

we find that the corresponding eigenvalues of $a_{1}$ and $a_{0}$ are determined by

$$
\left(2 a_{1}+45\right)^{2}\left(a_{1}+14\right)=0, \quad\left(a_{0}-18\right)\left(4 a_{0}^{2}-200 a_{0}+2423\right)=0,
$$

where we have made the simplifying assumptions $p=1, q=1, \ell=1$.

Note further that if we perform a gauge transformation in the nonseparable Cartesian coordinates to try to find polynomial solutions in $s_{1}^{2}, s_{2}^{2}$, we will obtain exactly the same exponential 
phase factor as the product of the phase factors in $u$ and in $v$. This means that the possible polynomial solutions in $s_{1}^{2}, s_{2}^{2}$ for a fixed energy eigenspace correspond exactly with the polynomial solutions symmetric in $u$ and $v$. The polynomial energy eigenspace is degenerate but the diagonalization of $a_{0}$ breaks the degeneracy. For a dramatic example of similar behavior on the $n$-sphere see Ref. 26.

\section{CONCLUSION}

We have demonstrated for a large class of superintegrable potentials that the method of separation of variables can be used to solve the corresponding Schrödinger equation in a specific form of elliptical coordinates. In these coordinates we have demonstrated that finite polynomial solutions are possible up to multiplication by an explicit function. This provides a generalization for the property of quasiexact solvability as given by Ushveridze. ${ }^{12}$ It also consolidates further the close connection with classical separation of variable concepts of families of polynomial solutions. In addition, the problem of finding all the algebraic equations determining the separation parameters has its natural place in the multivariable interpretation of these equations. The very fact that these polynomial solutions arise from superintegrable systems and that these systems have polynomial algebras associated with them implies that there are relations among the various solutions of the Schrödinger equation. This is a matter that we will pursue.

${ }^{1}$ E. G. Kalnins, W. Miller, Jr., and G. S. Pogosyan, J. Math. Phys. 47, 033502 (2006).

${ }^{2}$ J. Friš, Ya. A. Smorodinskii, M. Uhlír, and P. Winternitz, Sov. J. Nucl. Phys. 4, 444 (1967).

${ }^{3}$ A. A. Makarov, Ya. A. Smorodinsky, Kh. Valiev, and P. Winternitz, Nuovo Cimento A 52, 1061 (1967).

${ }^{4}$ N. W. Evans, Phys. Rev. A 41, 5666 (1990).

${ }^{5}$ C. Grosche, G. S. Pogosyan, and A. N. Sissakian, Fortschr. Phys. 43, 453 (1995); Phys. Part. Nucl. 27, 244 (1996); Phys. Part. Nucl. 28, 486 (1997).

${ }^{6}$ E. G. Kalnins, J. M. Kress, W. Miller, Jr., and G. S. Pogosyan, J. Phys. A 34, 4705 (2001).

${ }^{7}$ E. G. Kalnins, J. M. Kress, and W. Miller, J. Math. Phys. 46, 053509 (2005).

${ }^{8}$ E. G. Kalnins, J. M. Kress, and W. Miller, J. Math. Phys. 46, 053510 (2005).

${ }^{9}$ E. G. Kalnins, J. M. Kress, and W. Miller, J. Math. Phys. 46, 103507 (2005).

${ }^{10}$ E. G. Kalnins, J. M. Kress, and W. Miller, J. Math. Phys. 47, 043514 (2006).

${ }^{11}$ E. G. Kalnins, J. M. Kress, and W. Miller, J. Math. Phys. 47, 093501 (2006).

${ }^{12}$ A. G. Ushveridze, Quasi-exactly Solvable Models in Quantum Mechanics (IOP, Bristol, 1993).

${ }^{13}$ E. Magyari, Phys. Lett. 81A, 116 (1981).

${ }^{14}$ C. M. Bender and S. Boettcher, J. Phys. A 31, L273 (1998).

${ }^{15}$ C. M. Bender, Contemp. Phys. 466, 277 (2005).

${ }^{16}$ M. Znojil, J. Phys. A 32, 4563 (1999).

${ }^{17}$ M. Znojil, J. Phys. A 33, 4203 (2000).

${ }^{18}$ M. Znojil, J. Phys. A 33, 6825 (2000).

${ }^{19}$ P. Letourneau and L. Vinet, Ann. Phys. (N.Y.) 243, 144 (1995).

${ }^{20}$ E. G. Kalnins, J. M. Kress, W. Miller, Jr., and G. S. Pogosyan, Phys. At. Nucl. (to be published).

${ }^{21}$ E. T. Whittaker and G. N. Watson, A Course of Modern Analysis (Cambridge University Press, Cambridge, 1927), Vol. II.

${ }^{22}$ E. G. Kalnins, G. Williams, W. Miller, Jr., and G. S. Pogosyan, J. Math. Phys. 40, 708 (1999).

${ }^{23}$ A. R. Ronveaux, Heun's Differential Equation (Oxford University Press, New York, 1995).

${ }^{24}$ E. G. Kalnins, Separation of Variables for Riemannian Spaces of Constant Curvature, Pitman, Monographs and Surveys in Pure and Applied Mathematics (Longman, Essex, England, 1986).

${ }^{25}$ E. G. Kalnins, W. Miller, Jr., and G. J. Reid, Proc. R. Soc. London, Ser. A 394, 183 (1984).

${ }^{26}$ E. G. Kalnins, W. Miller, Jr., and M. Tratnik, SIAM J. Math. Anal. 22, 272 (1991). 